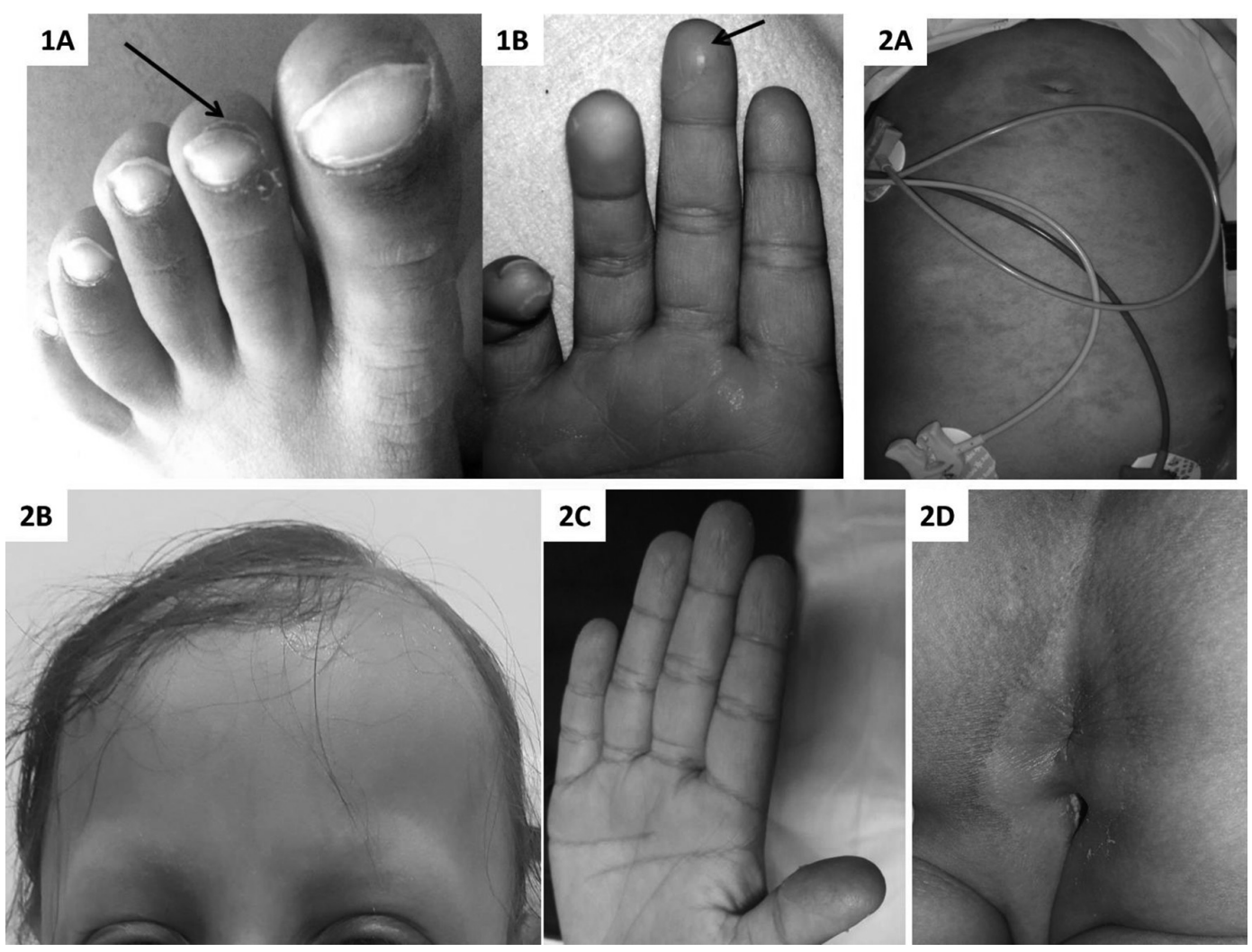

Abstract 18 Figure 1 (1A) Periungual skin peeling in the 2nd toe of left foot and the middle finger of the right hand (1B) in first child; (2A) Erythematous maculopapular rash on trunk in second child; (2B) diffuse alopecia with frontal baldness in second child; (2C) periungual skin peeling in all digits of right hand of second child; (2D) characteristic perianal skin peeling in second child.

context of $\mathrm{KD}$. We report 2 children who concomitantly had SLE and KD.

Methods Patient 1: A 5-year-old boy presented with fever of 2 months. He was irritable, he had malar rash, generalized erythematosus maculopapular rash, oral ulcers, red cracked lips, conjunctival injection, cervical adenopathy and hepatomegaly. Investigations showed anemia, leucopenia, elevated erythrocyte sedimentation rate (ESR) and high C-reactive protein (CRP). He had hypocomplimentemia; positive antinuclear antibody (ANA) (4+diffuse pattern) and high antidsDNA antibody titre $(1560 \mathrm{IU} / \mathrm{mL})$. Diagnosis of SLE was made. One week later he was brought with typical periungual peeling of skin in fingers and toes (figure 1). Investigations showed anemia (haemoglobin- $83 \mathrm{gm} / \mathrm{L}$ ), thrombocytosis $(510 \times 109 / \mathrm{L})$, persistently elevated ESR $(61 \mathrm{~mm}$ in 1 st hour) and high CRP $(16 \mathrm{mg} / \mathrm{L})$. So diagnosis of concomitant $\mathrm{KD}$ was considered.

Patient 2: A 8 year-old-girl presented with diffuse non scarring alopecia for 2 years and fever for 3 weeks. On examination, she had pallor; red cracked lips; palmar erythema; diffuse alopecia (figure1); generalized lymphadenopathy; hepatomegaly. Investigations revealed anaemia, lymphocytopenia, elevated ESR and CRP, high serum pro-BNP levels. She had hypocomplimentemia; positive ANA (4+diffuse pattern) and positive anti-dsDNA antibody. A diagnosis of SLE was made. But, on day 5 of hospital stay she developed periungual and perianal peeling (figure 1), and chromonychia. On reviewing history, she also had redness of lips and tongue, conjunctival injection and generalized maculopapular rash 5 days prior to admission. Possibility of concomitant KD was considered.

Results Both children fulfilled the classification criteria for diagnosis of SLE and KD as well and time course of events was also very suggestive. Patient 1 was administered intravenous immunoglobulin (IVIG) ( $\mathrm{gm} / \mathrm{kg})$, following which his irritability subsided. Patient 2 was also given IVIG $(2 \mathrm{gm} / \mathrm{kg})$. Both patients were discharged on oral prednisolone, hydroxychloroquine and low dose aspirin. Aspirin was stopped after 6 weeks as echocardiography was normal. Both continue to remain well on follow-up.

Conclusions While overlap of childhood SLE with other rheumatological disorders has been described, concomitant occurrence of SLE and $\mathrm{KD}$ is an extremely rare. Two or more rheumatologic diseases may coexist at the same time and one must always be vigilant.

Funding Source(s): No funding source.

\section{EVALUATING MEANINGFUL CHANGE IN DISEASE ACTIVITY AS A CLINICAL EFFICACY MEASURE FOR CLINICAL TRIALS IN CUTANEOUS LUPUS ERYTHEMATOSUS}

${ }^{1}$ Srita Chakka*, 'Rebecca L Krain, ${ }^{1}$ Sarah Ahmed, ${ }^{1}$ Rui Feng, ${ }^{2}$ Victoria P Werth. 'University of Pennsylvania; ${ }^{2}$ Perelman School of Medicine, University of Pennsylvania

\subsection{6/lupus-2019-|sm.19}

Background To date, there are no approved treatments for cutaneous lupus erythematous (CLE), a disease known to significantly burden a patients quality of life (QoL). Clinical trials are important for the advancement of treatments for CLE and trial outcome measures should reflect clinically meaningful improvement in disease activity and its effect on QoL. Currently, trials use an efficacy measure of 50\% improvement in 
Abstract 19 Table 1 Difference and percent change needed in CLASI-A scores to predict meaningful improvement in Skindex-29 subscales (Emotions and Symptoms) in patients with a range of initial CLASI-A scores

\begin{tabular}{|c|c|c|c|}
\hline \multicolumn{2}{|c|}{ Initial CLASI-A $\geq 8$} & \multirow{2}{*}{$\begin{array}{l}\text { Percent Change } \\
\text { in CLASI-A }\end{array}$} & \multirow[t]{2}{*}{ p-value } \\
\hline$n=90$ & Skindex-29 Subscale & & \\
\hline Emotions & & 42.06 & $<0.0001$ \\
\hline Symptoms & & 30.97 & $<0.0001$ \\
\hline \multicolumn{4}{|c|}{ Mild Disease Activity (CLASI-A 4-9) } \\
\hline$n=49$ & Skindex-29 Subscale & & \\
\hline Emotions & & 75.04 & 0.0004 \\
\hline Symptoms & & 110.0 & 0.0510 \\
\hline \multicolumn{4}{|c|}{ Moderate Disease Activity (CLASI-A 10-20) } \\
\hline$n=46$ & Skindex-29 Subscale & & \\
\hline Emotions & & 49.11 & 0.0050 \\
\hline Symptoms & & 33.96 & $<0.0001$ \\
\hline \multicolumn{4}{|c|}{ Severe Disease Activity (CLASI-A>20) } \\
\hline$n=31$ & Skindex-29 Subscale & & \\
\hline Emotions & & 25.42 & 0.0010 \\
\hline Symptoms & & 18.90 & 0.0080 \\
\hline Initial CLA & $\mathrm{I}-\mathrm{A} \geq 8$ & Difference in CLASI-A & p-value \\
\hline$n=90$ & Skindex-29 Subscale & & \\
\hline Emotions & & 6.872 & $<0.0001$ \\
\hline Symptoms & & 5.107 & $<0.0001$ \\
\hline \multicolumn{4}{|c|}{ Mild Disease Activity (CLASI-A 4-9) } \\
\hline$n=49$ & Skindex-29 Subscale & & \\
\hline Emotions & & 4.630 & 0.0006 \\
\hline Symptoms & & 5.722 & 0.0235 \\
\hline \multicolumn{4}{|c|}{ Moderate Disease Activity (CLASI-A 10-20) } \\
\hline$n=46$ & Skindex-29 Subscale & & \\
\hline Emotions & & 7.415 & 0.0067 \\
\hline Symptoms & & 5.051 & $<0.0001$ \\
\hline \multicolumn{4}{|c|}{ Severe Disease Activity (CLASI-A>20) } \\
\hline$n=31$ & Skindex-29 Subscale & & \\
\hline Emotions & & 6.541 & 0.0002 \\
\hline Symptoms & & 4.426 & 0.0011 \\
\hline
\end{tabular}

disease activity, defined by the Cutaneous Lupus Disease Area and Severity Index (CLASI) score for activity (CLASI-A), in patients with an initial CLASI-A 10. We aim to define the degree of improvement in disease activity needed to have a meaningful impact on QoL, an important variable in the design and interpretation of future clinical trials.

Methods This study included 126 patients seen at the Autoimmune Skin Disease Clinic at the Hospital of the University of Pennsylvania who participate in a longitudinal research database. Patients with mild, moderate or severe initial CLASI-A were analyzed separately, with further stratification of patients with mild initial activity. A linear regression model was used to calculate the percent change and difference needed in CLASI-A to have an important impact on QoL, defined as a 9.38-point and a 7.37-point improvement in the Emotions and Symptoms subscales of Skindex-29, respectively.

Results In patients with an initial CLASI-A 8, an improvement of $42.1 \%$ or 7 -points and an improvement of $31.0 \%$ or 5 points in disease activity is associated with a meaningful improvement in the Emotions and the Symptoms subscales, respectively. For both subscales, patients with increasingly severe initial disease required a smaller percent change in CLASI-A to predict a meaningful change in QoL (table 1).

Conclusions We find that using a CLASI-A 8 for trial entry allows for the inclusion of patients with milder disease for whom improvement of CLASI-A by 50\% results in a meaningful impact on QoL, as determined by the Emotions and Symptoms subscales of Skindex-29. In patients with CLASI-A 8 , a decrease in activity by seven and five-points is not only a clinically significant improvement but also indicates a meaningful impact on the Emotions and Symptoms subscales, respectively. For trials enrolling patients with CLASI-A 20, we recommend stratifying patients by disease severity, as a smaller percent change in activity in patients with severe disease can predict meaningful improvement in QoL. Our findings establish appropriate trial endpoints by determining clinically significant change in disease activity associated with meaningful changes in patients QoL.

Funding Source(s): NIH/NIAMS 1R01AR071653 - 01A1

\section{ANTI-NEUTROPHIL CYTOPLASMIC ANTIBODIES IN LUPUS NEPHRITIS}

${ }^{1}$ May Choi ${ }^{*},{ }^{2}$ Ann E Clarke, ${ }^{3}$ Alex Chin, ${ }^{4}$ Michelle Jung, ${ }^{2}$ Claire Barber, ${ }^{4}$ Marvin Fritzler. ${ }^{1}$ Cumming School of Medicine, University of Calgary; ${ }^{2}$ Division of Rheumatology, Cumming School of Medicine, University of Calgary; ${ }^{3}$ University of Calgary, Calgary Laboratory Services; ${ }^{4}$ University of Calgary

\subsection{6/lupus-2019-Ism.20}

Background Anti-myeloperoxidase (MPO) antibodies have been shown to predict the development of proliferative lupus nephritis (LN) suggesting anti-neutrophil cytoplasmic antibodies (ANCA) may have a pathogenic and prognostic role in LN. This study compared the type of LN, renal function, and systemic lupus erythematosus (SLE)-related and antiphospholipid autoantibodies between LN patients who were ANCA (antiproteinase 3 (PR3) and anti-MPO antibodies) positive and negative.

Methods Patients fulfilling the ACR or SLICC Classification Criteria for SLE were enrolled in a local cohort. We retrospectively identified patients with Class 2, 3, 4, or $5 \mathrm{LN}$ on renal biopsy who also had an ANCA, plasma creatinine, and urine protein creatinine ratio (UPCR) at time of biopsy. ANCA by IIF was performed on ethanol and formalin-fixed polymorphonuclear leukocytes and a HEp-2 cell biochip (EuroPattern, Euroimmun $\mathrm{GmbH}$, Luebeck, Germany) while antibodies to MPO and PR3 were determined by multiplex immunoassay (Bio-Rad, Hercules, CA: BioPlex 2200, cutoff $\geq 2 \mathrm{KEU} / \mathrm{L})$. Using sera collected at enrollment, SLErelated autoantibodies (dsDNA, Sm, U1RNP, Sm, Ro52/ TRIM21, Ro60/SSA, SS-B/La, Scl-70, Jo-1, RiboP, PCNA, PM/ $\mathrm{Scl}$ ) were performed by laser bead immunoassay (Euroimmune), lupus anticoagulant by tissue thromboplastin inhibition test and dilute Russell viper venom time, and anti-cardiolipin IgG and anti-2 glycoprotein-1 IgG by ELISA. Comparisons were performed with Fishers exact or Mann-Whitney U.

Results 23 SLE patients with LN were included; $82.6 \%$ were female. Most patients $(20 / 23,87.0 \%)$ were ANCA positive by IIF while only $5 / 23(21.7 \%)$ had antibodies to MPO $(3 / 23$, $13.0 \%)$ or both $\mathrm{MPO}$ and PR3 (2/23, 8.7\%). Anti-MPO/PR3 positive patients had p-ANCA $(2 / 5,40 \%)$ or an atypical pattern $(3 / 5,60 \%)$ on ANCA IIF. When comparing anti-MPO/ PR3 positive (5) to negative (18) patients, there was no difference in LN class, creatinine, UPCR, or the presence of SLErelated autoantibodies. Anti-cardiolipin IgG antibodies were more common in anti-MPO/PR3 positive patients $(60.0 \%$ vs $5.6 \%, \mathrm{p}=0.021)$, while a nuclear pattern on ANCA IIF was more common in anti-MPO/PR3 negative patients $(55.6 \%$ vs. 